\title{
Circular RNA circZNF652 is overexpressed in osteoarthritis and positively regulates LPS-induced apoptosis of chondrocytes by upregulating PTEN
}

\section{Xuefeng Yuan}

Huazhong University of Science and Technology

\section{Yingchi Zhang}

Huazhong University of Science and Technology

\section{Cong Cai}

Huazhong University of Science and Technology

Chaoxu Liu

Huazhong University of Science and Technology

Jie Xie

Huazhong University of Science and Technology

Chengla Yi ( $\nabla$ chenglaYiSurgery@163.com )

Huazhong University of Science and Technology

\section{Research}

Keywords: osteoarthritis, circZNF652, PTEN, apoptosis

Posted Date: September 2nd, 2020

DOI: https://doi.org/10.21203/rs.3.rs-58776/v1

License: (c) (1) This work is licensed under a Creative Commons Attribution 4.0 International License.

Read Full License

Version of Record: A version of this preprint was published at Autoimmunity on July 15 th, 2021 . See the published version at https://doi.org/10.1080/08916934.2021.1951716. 


\section{Abstract \\ Background}

Circular RNA circZNF652 promotes LPS-induced inflammation, which contributes to the development of osteoarthritis (OA), indicating the potential involvement of CRNDE in OA. This study was carried to explore the involvement of circZNF652 in OA.

\section{Methods}

RT-qPCR was performed to analyze the expression of circZNF652 and PTEN mRNA in synovial fluid samples from 60 OA patients and 60 healthy controls. Correlations between circZNF652 and PTEN mRNA were analyzed by Pearson's correlation coefficient. Overexpression and siRNA silencing of circZNF652 were achieved in chondrocytes, followed by performing RT-qPCR and Western blot to analyze the expression of PTEN. The role of circZNF652 and PTEN in regulating the apoptosis of chondrocytes induced by LPS was analyzed by cell apoptosis assay.

\section{Results}

We found that circZNF652 was overexpressed in OA and positively correlated with PTEN mRNA. In chondrocytes, circZNF652 overexpression increased the expression of PTEN, and circZNF652 siRNA silencing decreased the expression of PTEN. Moreover, circZNF652 and PTEN positively regulated the apoptosis of chondrocytes induced by LPS. PTEN overexpression reversed the inhibitory effects of circZNF652 siRNA silencing on cell apoptosis.

\section{Conclusion}

CircZNF652E is overexpressed in OA and positively regulates LPS-induced apoptosis of chondrocytes by upregulating PTEN.

\section{Introduction}

Osteoarthritis (OA) as the major cause of disability and chronic pain is the main subtype of arthritis that affects about $13 \%$ of females and $10 \%$ of males worldwide [1]. OA occurs when the protective cartilage wears down during aging or after injuries [2]. Theoretically OA can cause irreversible damages to all joints, while the most commonly affected sites are knees, hips, hands and spine [2,3]. At present, the treatment of OA mainly focus on the relief of symptoms, such as the use of Acetaminophen to reduce moderate or mild pain, while current available medications may not work in severe cases [4, 5]. In addition, full recovery is still rare in clinical practices [6]. Therefore, novel treatment approaches are needed. 
Aging is the major risk factor for $O A$, while aging is not sufficient for the occurrence and development of $\mathrm{OA}$. In effect, the development and progression require the involvement of multiple molecular signaling pathways, such as Notch, NF-KB and PTEN [7, 8]. In effect, some key molecular players have been proven as potential targets for targeted OA therapy, which can be used in clinical practices to treat OA by regulating the network of gene expression $[9,10]$. Despite the lacking of protein-coding capacity, circular RNAs (circRNAs) regulate gene expression to participate in human diseases including OA by regulating the expression of key genes [11-13]. Therefore, circRNAs are promising targets for targeted OA therapy. However, the function of cirRNAs in OA remains hardly known. It has been reported that circZNF652 promote LPS-induced inflammatory responses [14], which contributes to OA [15]. Our preliminary microarray analysis revealed the altered expression of circZNF652 in OA and its close correlation with PTEN, a key player in OA [8]. This study was performed to explore the potential crosstalk between circZNF652 and PTEN in OA.

\section{Materials And Methods}

\section{OA patients and controls}

Research subjects of this study included 60 OA patients (40 males and 20 females) and 60 healthy controls (40 males and 20 females). All patients and controls provided informed consent. All patients were admitted to Tongji Hospital, Tongji Medical College, Huazhong University of Science and Technology from May 2018 to May 2020. Age range of OA patients was 41 to 60 years, with a median of 51 years. Age range of healthy controls was 41 to 60 years, with a median of 52 years. All patients were excluded from initiated therapy with 3 months before admission and other severe clinical disorders. No recurrent cases were enrolled, and all patients were newly diagnosed cases. Among the 60 OA patients, hip was affected in 31 cases and knee was affected in 29 cases. Healthy controls showed normal physiological functions after systemic physiological exams at the aforementioned hospital. Ethics Committee of aforementioned hospital approved this study.

\section{Synovial fluid and chondrocytes}

Prior to therapy, a syringe was used to extract synovial fluid $(2 \mathrm{ml})$ from the affected sites of patients. To perform control experiment, synovial fluid was also collected from the corresponding sites of healthy controls (31 cases of hip and 29 cases of knee). Liquid nitrogen storage was performed prior to the subsequent assays.

Primary chondrocytes from Sigma-Aldrich (Cat\# 4020A-05A, USA) were included in this study to serve as the cell model of OA. These chondrocytes were from an adult with OA. Cell culture was performed following manufacturer's instructions. In cases of LPS treatment, cells were cultivated in medium supplemented with 0, 2, 4, 6, 8 and $10 \mu \mathrm{g} / \mathrm{ml}$ LPS for further $48 \mathrm{~h}$ prior to the subsequent experiments.

\section{Vectors, siRNAs and transfections}


With pcDNA3.1 vector (Invitrogen) and pcDNA3.1(+) CircRNA Mini Vector (Addgene) as backbone, expression vectors of circZNF652 and PTEN were constructed, respectively. SiRNA negative control (NC) and circZNF652 siRNA were purchased from Invitrogen. Chondrocytes (108) at around 80\% confluence were subjected to expression vector $(1 \mu \mathrm{g})$ or NC miRNA $(40 \mathrm{nM})$ transfection using lipofectamine 2000 (Invitrogen). NC experiments were performed by transfecting the same amount of empty vector or NC siRNA into the same number of cells. In all transfections, control (C) cells were untransfected cells. In fresh medium cells were cultivated in fresh

medium for $48 \mathrm{~h}$ prior to the subsequent assays.

\section{RNA exrtraction}

Isolation of RNA from synovial fluid and chondrocytes was performed using RNAzol (Sigma-Aldrich), followed by genomic DNA removal performed by DNase I digestion at $37^{\circ} \mathrm{C}$ for $2 \mathrm{~h}$. RNA integrity was analyzed by $5 \%$ urea-PAGE gel electrophoresis.

\section{RT-qPCR}

RNA purifty was analyzed by measuring OD 260/280 rations. RNA samples with an OD 260/280 ratio close to 2.0 (pure RNA) were subjected to reverse transcriptions using SS-IV-RT system (Invitrogen). SYBR ${ }^{\circledR}$ Green Quantitative RT-qPCR Kit (Sigma-Aldrich) was used to perform all qPCRs with GAPDH as an internal control to determine the expression of circZNF652 and PTEN mRNA. Three technical replicates were included in each reaction. Ct value normalizations were performed using $2^{-\triangle \triangle C T}$ method.

\section{Western-blot}

PIPA solution (Invitrogen) and BCA assay (Invitrogen) were used to extract and quantify protein from chondrocytes. In boiling water RNA samples were incubated for $12 \mathrm{~min}$ to achieve protein denaturation. Electrophoresis was performed using $10 \%$ SDS-PAGE gel, followed by using PVDF membranes to transfer proteins. Following blocking in $5 \%$ fat-free milk for 2h, GAPDH (1: 1600, ab37168, Abcam) and PTEN (1: 1600, ab31392, Abcam) primary rabbit antibodies were used to incubate membranes overnight at $4{ }^{\circ} \mathrm{C}$. After that, anti-rabbit IgG-HRP (1:1600, MBS435036, MyBioSource) secondary antibody was used to incubate membranes for $2 \mathrm{~h}$. ECL (Sigma-Aldrich) was used to develop signals and Image J v1.46 software was used for data normalizations.

\section{Cell apoptosis analysis}

Chondrocytes with transfections were subjected to cell apoptosis analysis through cell apoptosis assay. A 6-well cell culture plate was used to cultivate chondrocytes (20,000 cells per well) in medium containing $10 \mu \mathrm{g} / \mathrm{ml}$ LPS for further $48 \mathrm{~h}$ at $37^{\circ} \mathrm{C}$. Following $0.25 \%$ trypsin digestion, propidium iodide (PI, Dojindo, Japan) and Annexin V-FITC (Dojindo, Japan) staining was performed, followed by flow cytometry to analyze cell apoptosis. 


\section{Statistical analysis}

Expression levels of circZNF652 and PTEN mRNA in synovial fluid samples from both OA patients $(n=60)$ and healthy controls $(n=60)$ were expressed as average values of three technical replicates and unpaired $t$ test was used for data comparison. Data of multiple cell transfection groups and LPS treatment groups was expressed as mean +/-SD values of three biological replicates, and ANOVA Tukey's test was used for data comparisons. Correlations were analyzed by Pearson's correlation coefficient. $P<0.05$ was deemed statistically significant.

\section{Results}

\section{CircZNF652 and PTEN mRNA were both overexpressed in OA and they were closely correlated}

CircZNF652 and PTEN mRNA expression in synovial fluid samples from both OA patients $(n=60)$ and healthy controls $(n=60)$ was analyzed by RT-qPCR. Unpaired t test analysis revealed that CircZNF652 (Fig.1A) and PTEN mRNA (Fig.1B) were significantly overexpressed in OA samples compared to control samples $(p<0.01)$. Pearson's correlation coefficient was performed to analyze the correlations between CircZNF652 and PTEN mRNA across both OA and control samples. It was observed that CircZNF652 and PTEN mRNA were positively and significantly correlated with each other across OA samples (Fig.1C), but not control samples (Fig.1D), suggesting the existence of the specific crosstalk between them in OA.

\section{LPS treatment increased the expression of both circZNF652 and PTEN mRNA in chondrocytes in dose- dependent manner}

Chondrocytes were cultivated in medium supplemented with $0,2,4,6,8$ and $10 \mu \mathrm{g} / \mathrm{ml}$ LPS for further $48 \mathrm{~h}$ prior to the determination of the expression of circZNF652 and PTEN mRNA by RT-qPCR. It was observed that expression levels of circZNF652 (Fig.2A) and PTEN mRNA (Fig.2B) were increased by LPS treatment in a dose-dependent manner. Therefore, circZNF652 and PTEN mRNA may participate in OA through a LPS-dependent pathway.

\section{CircZNF652 positively regulated the expression of PTEN in chondrocytes}

To explore the crosstalk between circZNF652 and PTEN, chondrocytes were transfected with either circZNF652 or PTEN expression vector, or circZNF652 siRNA, followed by the confirmation of transfections by RT-qPCR (Fig.3A, p<0.05). CircZNF652 overexpression increased the expression of PTEN (Fig.3B, $p<0.05$ ), and circZNF652 siRNA silencing decreased the expression of PTEN (Fig.3C, $p<0.05$ ). In contrast, PTEN overexpression failed to significantly alter the expression of circZNF652 (Fig.3D). Therefore, circZNF652 is likely an upstream activator or PTEN in chondrocytes.

\section{CircZNF652 positively regulated the apoptosis of chondrocytes induced by LPS through PTEN}

Chondrocytes were cultivated in medium containing $10 \mu \mathrm{g} / \mathrm{ml} \mathrm{LPS} \mathrm{for} \mathrm{further} 48 \mathrm{~h}$ at $37^{\circ} \mathrm{C}$, followed by the analysis of cell apoptosis by cell apoptosis assay. It was observed that circZNF652 and PTEN 
overexpression increased cell apoptosis, while circZNF652 siRNA silencing decreased cell apoptosis. Moreover, PTEN overexpression reversed the inhibitory effects of circZNF652 siRNA silencing on cell apoptosis (Fig.4, p<0.05).

\section{Discussion}

The differential expression of circZNF652 in OA and its role in regulating cell apoptosis were explored in this study. We found that circZNF652 was overexpressed in OA and it could positively regulate PTEN to promote the apoptosis of chondrocytes induced by LPS.

OA is essentially a type of inflammatory disease, in which LPS-induced inflammation plays an important role [16]. In a recent study, Liu et al. reported that LPS treatment increased the expression of circZNF652 in WI-38 cells, which are fibroblasts derived from lung tissue, and the knockdown of circZNF652 suppresses inflammatory damages caused by LPS treatment [14]. Consistently, our study showed that LPS treatment also increased the expression of circZNF652 in chondrocytes derived from an adult with OA. Therefore, LPS may induce circZNF652 expression in different types of cells.

In this study we showed that circZNF652 was significantly overexpressed in OA. Chondrocytes, which are the only mature cells observed in healthy cartilage, produce collagen and proteoglycans to maintain the cartilaginous matrix [17]. In OA, increased apoptosis of chondrocytes contributes to the development of disease [17]. In addition, cell apoptosis analysis showed that circZNF652 positively regulated the apoptosis of chondrocytes induced by LPS treatment. Therefore, circZNF652 may promote OA by increasing the apoptosis of chondrocytes, and siRNA silencing of circZNF652 may serve as a potential therapeutic target for $\mathrm{OA}$.

The main role of PTEN is to suppress PI3K/Akt pathway, which is the main cell survival pathway, to induce cell apoptosis [18]. In OA, PTEN was overexpressed to promote disease development by increasing cell apoptosis [8]. Consistently, our study revealed the upregulation of PTEN in OA and its enhancing effects on the apoptosis of chondrocytes induced by LPS. It has been well established that the main role of circRNAs is to regulate gene expression at transcriptional and/or translational levels [11]. In this study we showed that circZNF652 overexpression increased PTEN expression at both mRNA and protein levels. Therefore, circZNF652 overexpression may at least promote the transcription of PTEN. However, circZNF652 and PTEN were only positively correlated only across OA samples, not control samples. Therefore, certain pathological factors may mediate the interaction between them.

\section{Conclusion}

circZNF652 is overexpressed in OA. In addition, circZNF652 may promote the apoptosis of chondrocytes induced by LPS by positively regulating the expression of PTEN.

\section{Declarations}




\section{Ethics approval and consent to participate}

This study was approved by Tongji Hospital, Tongji Medical College, Huazhong University of Science and Technology.

\section{Consent for publication}

Informed consent was obtained from all patients.

\section{Availability of data and materials}

The datasets used and/or analysed during the current study are available from the corresponding author on reasonable request.

\section{Competing interests}

The authors declare that they have no competing interests.

\section{Funding}

No funding was received.

\section{Authors' contributions}

$\mathrm{XY}$ designed the experiments. $\mathrm{YZ}, \mathrm{CC}$ and $\mathrm{CL}$ performed the experiments. JX analyzed the data. CL wrote the draft paper. All authors read and approved the final manuscript.

\section{Acknowledgements}

Not applicable.

\section{References}

1. Glyn-Jones S, Palmer AJR, Agricola R, et al. Osteoarthritis Lancet. 2015;386(9991):376-87.

2. Vina ER, Kwoh CK. Epidemiology of osteoarthritis: literature update. Curr Opin Rheumatol. 2018;30(2):160-7.

3. Eaton $\mathrm{CB}$, Roberts $\mathrm{M}$, Driban JB, et al. Multiple joint osteoarthritis in the osteoarthritis initiative. Osteoarthritis Cartilage. 2019;27:100.

4. Gu YT, Chen J, Meng ZL, et al. Research progress on osteoarthritis treatment mechanisms. Biomed Pharmacother. 2017;93:1246-52.

5. Banks C, Hughes K, Simpkins C, et al. Osteoarthritis in Older Adults: Nonsteroidal Anti-Inflammatory Drugs Versus Opioids. Sr Care Pharm. 2019;34(10):674-7.

6. Bahl JS, Nelson MJ, Taylor M, et al. Biomechanical changes and recovery of gait function after total hip arthroplasty for osteoarthritis: a systematic review and meta-analysis. Osteoarthritis Cartilage. 
2018;26(7):847-63.

7. Saito T, Tanaka S. Molecular mechanisms underlying osteoarthritis development: Notch and NF-KB. Arthritis Res Ther. 2017;15(1):94. 19(.

8. Galasso O, Panza S, Santoro M, et al. PTEN elevation, autophagy and metabolic reprogramming may be induced in human chondrocytes during steroids or nutrient depletion and osteoarthritis. J Biol Regul Homeost Agents. 2015;29(Suppl 4):1-14.

9. Roman-Blas JA, Jimenez SA. NF-kB as a potential therapeutic target in osteoarthritis and rheumatoid arthritis. Osteoarthritis Cartilage. 2006;14(9):839-48.

10. Mobasheri A. The future of osteoarthritis therapeutics: targeted pharmacological therapy. Curr Rheumatol Rep. 2013;15(10):364.

11. Qu S, Yang X, Li X, et al. Circular RNA: a new star of noncoding RNAs. Cancer Lett. 2015;365(2):1418.

12. Yu CX, Sun S. An emerging role for circular RNAs in osteoarthritis. Yonsei Med J. 2018;59(3):349-55.

13. Xiao K, Xia Z, Feng B, et al. Circular RNA expression profile of knee condyle in osteoarthritis by illumina HiSeq platform. J Cell Biochem. 2019;120(10):17500-11.

14. Liu X, Zhao P, Ge W. Knockdown of circular RNA circZNF652 remits LPS-induced inflammatory damage by regulating miR-181a. Biofactors. 2019. doi:10.1002/biof.1606.

15. Scotece $\mathrm{M}$, Conde J, Abella V, et al. Oleocanthal inhibits catabolic and inflammatory mediators in LPS-activated human primary osteoarthritis (OA) chondrocytes through MAPKs/NF-KB pathways. Cell Physiol Biochem. 2018;49(6):2414-26.

16. Goldring MB, Otero M. Inflammation in osteoarthritis. Curr Opin Rheumatol. 2011;23(5):471-8.

17. Blanco FJ, Guitian R, Vázquez-Martul E, et al. Osteoarthritis chondrocytes die by apoptosis: a possible pathway for osteoarthritis pathology. Arthritis Rheum. 1998;41(2):284-9.

18. Yamada KM, Araki M. Tumor suppressor PTEN: modulator of cell signaling, growth, migration and apoptosis. J Cell Sci. 2001;114(13):2375-82.

\section{Figures}




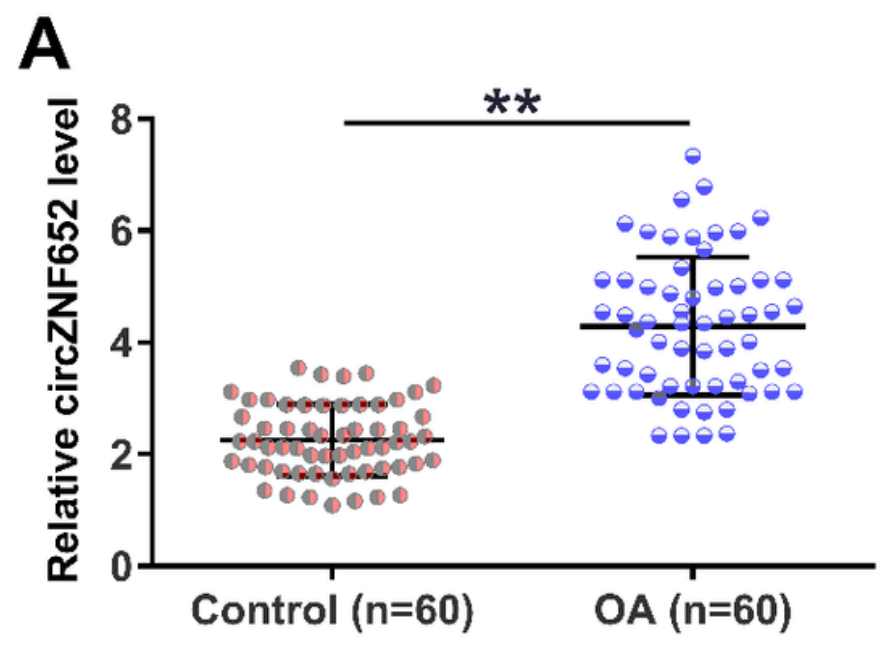

\section{B}

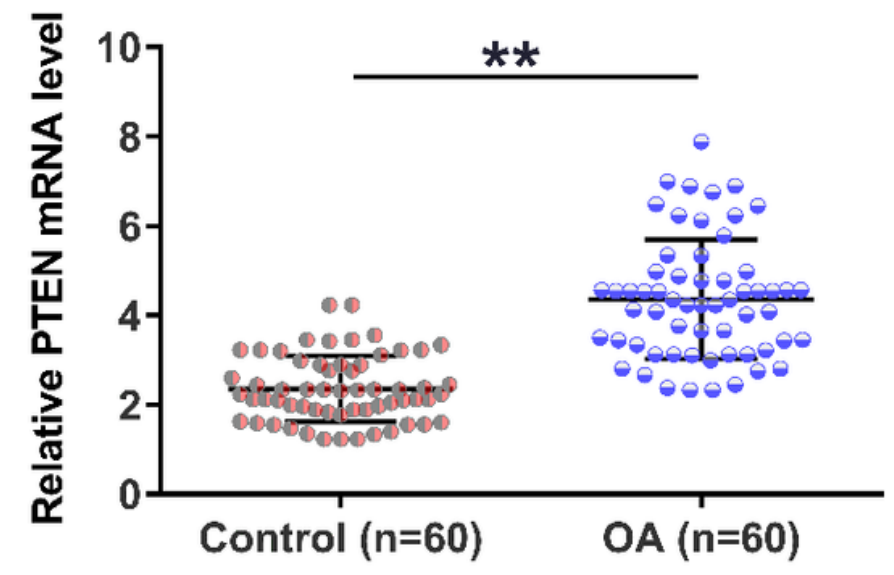

C
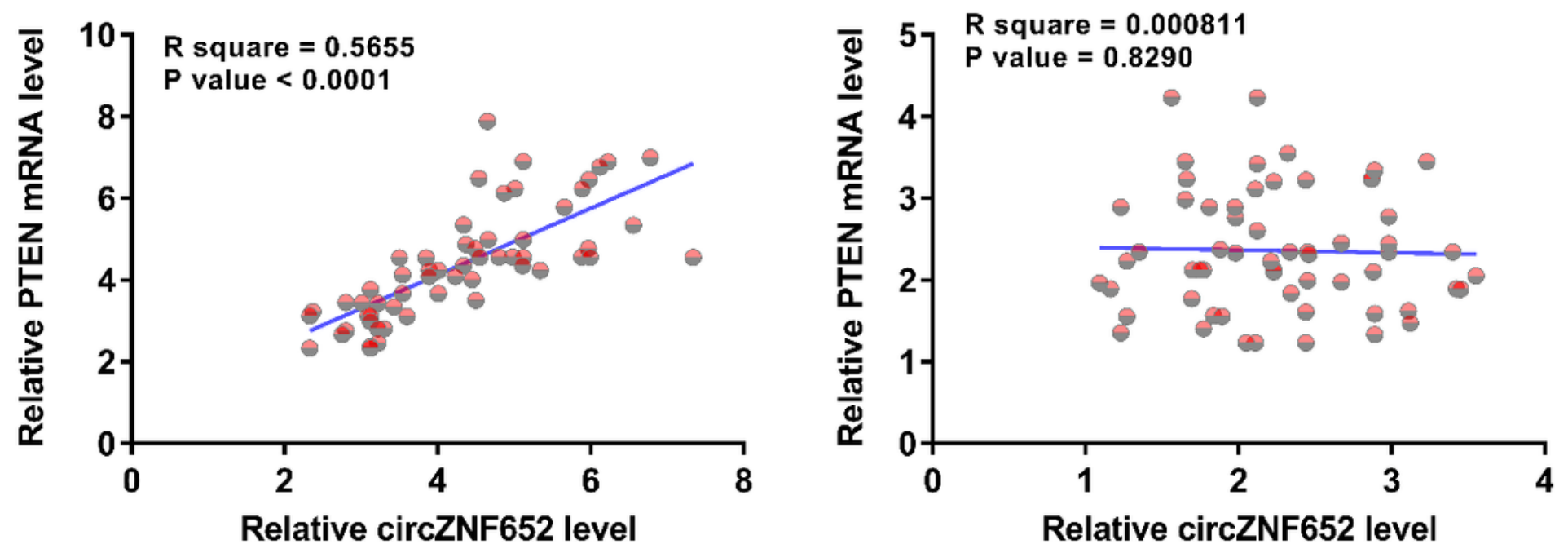

Figure 1

CircZNF652 and PTEN mRNA were both overexpressed in OA and they were closely correlated CircZNF652 and PTEN mRNA expression in synovial fluid samples from both OA patients $(n=60)$ and healthy controls $(n=60)$ was analyzed by RT-qPCR. Unpaired t test was performed to compare CircZNF652 (A) and PTEN mRNA (B) expression between OA samples and control samples. Expression levels of circZNF652 and PTEN mRNA in synovial fluid samples from both OA patients $(n=60)$ and healthy controls $(n=60)$ were expressed as average values of three technical replicates. ${ }^{\star \star}, p<0.01$. Pearson's correlation coefficient was performed to analyze the correlations between CircZNF652 and PTEN mRNA across both OA (C) and control (D) samples. 


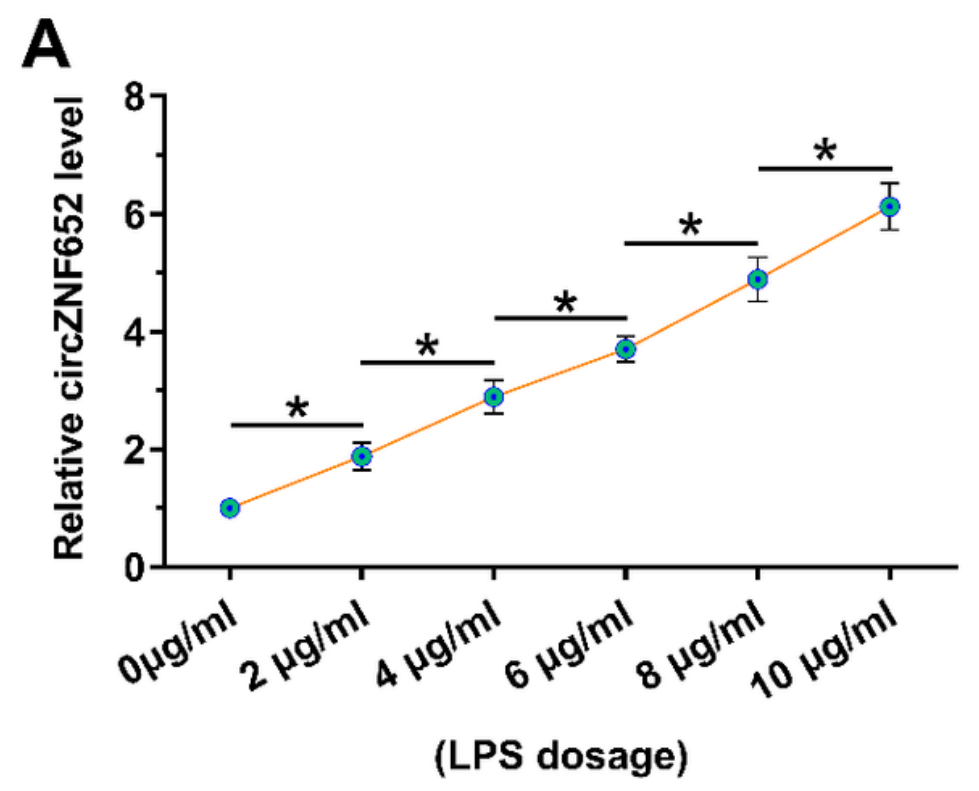

B

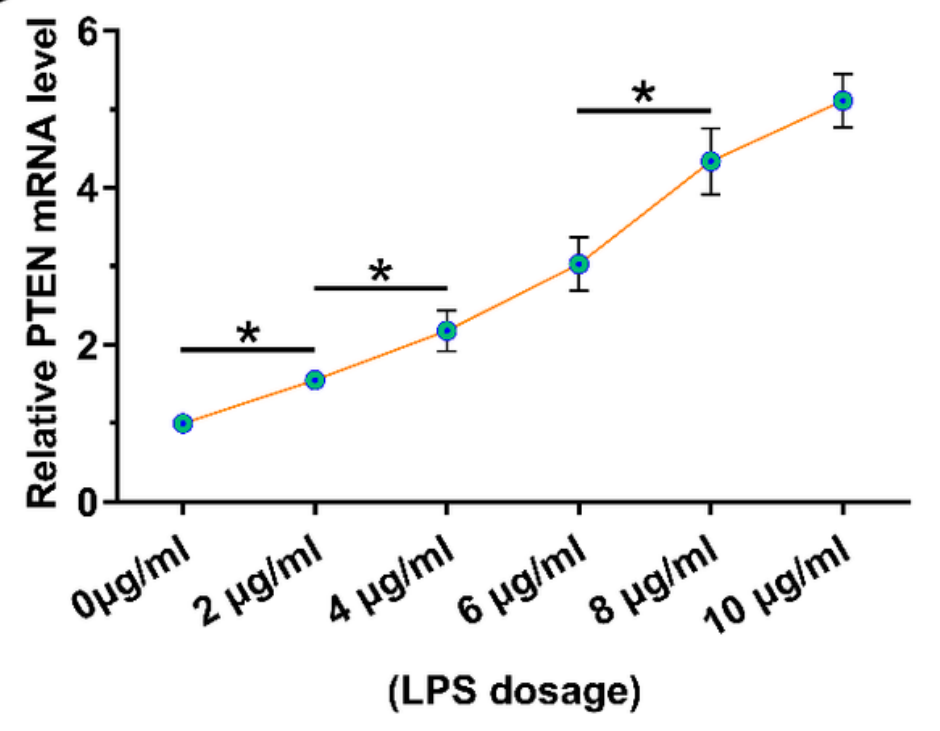

Figure 2

LPS treatment increased the expression of both circZNF652 and PTEN mRNA in chondrocytes in dosedependent manner Chondrocytes were cultivated in medium supplemented with $0,2,4,6,8$ and $10 \mu \mathrm{g} / \mathrm{ml}$ LPS for further $48 \mathrm{~h}$ prior to the determination of the expression of circZNF652 (A) and PTEN mRNA (B) by RT-qPCR. Data of multiple cell transfection groups and LPS treatment groups was expressed as mean $+/-$ $S D$ values of three biological replicates. ${ }^{*}, p<0.05$. 

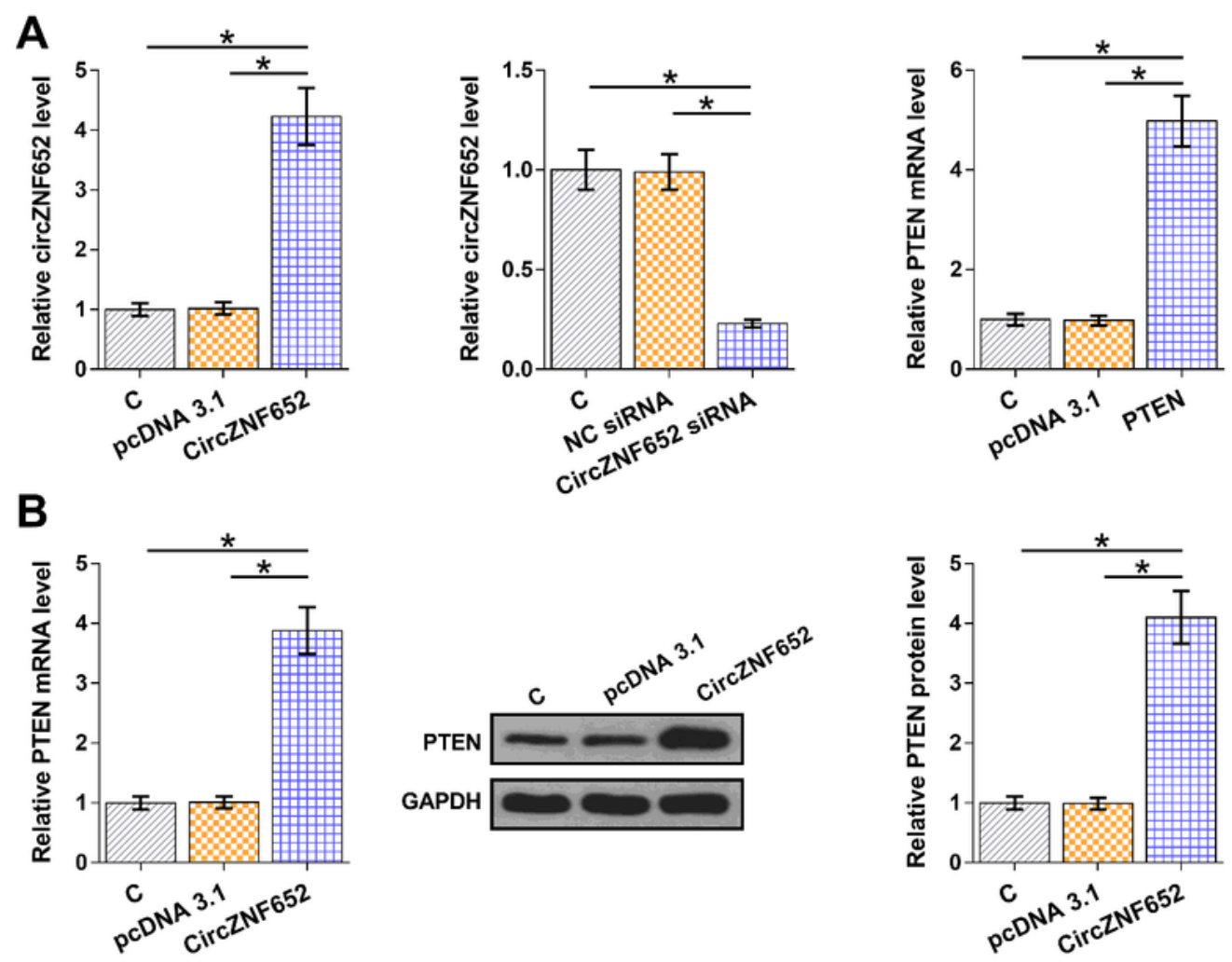

C
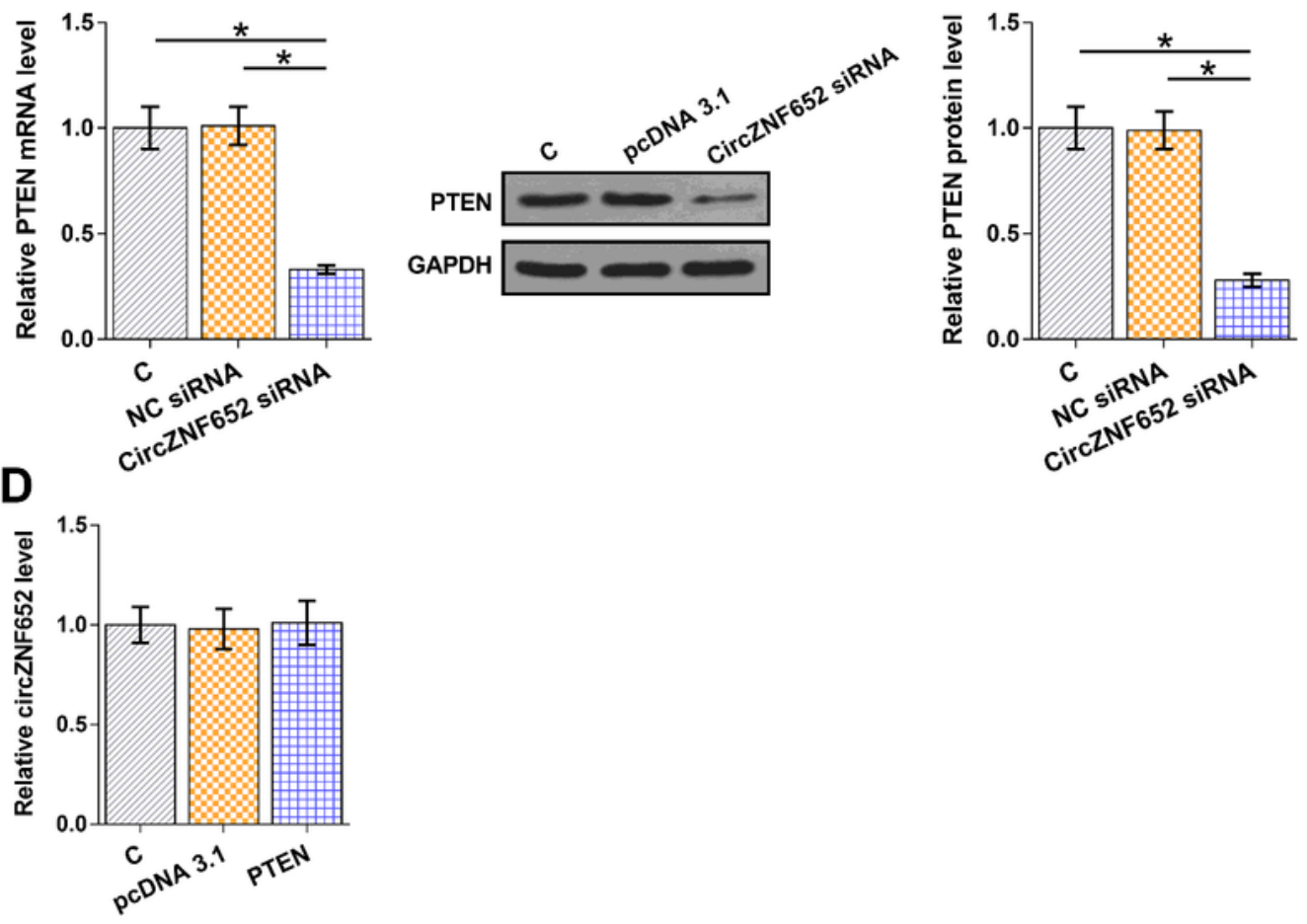

Figure 3

CircZNF652 positively regulated the expression of PTEN in chondrocytes To explore the crosstalk between circZNF652 and PTEN, chondrocytes were transfected with either circZNF652 or PTEN expression vector, or circZNF652 siRNA, followed by the confirmation of transfections by RT-qPCR (A). The effects of circZNF652 overexpression (B) and siRNA silencing (C) on the expression of PTEN were explored by RT-qPCR and Western blot (D). The effects of PTEN overexpression on circZNF652 were 
explored by RT-qPCR. Data of multiple cell transfection groups and LPS treatment groups was expressed as mean $+/$ - SD values of three biological replicates. ${ }^{*}, p<0.05$.

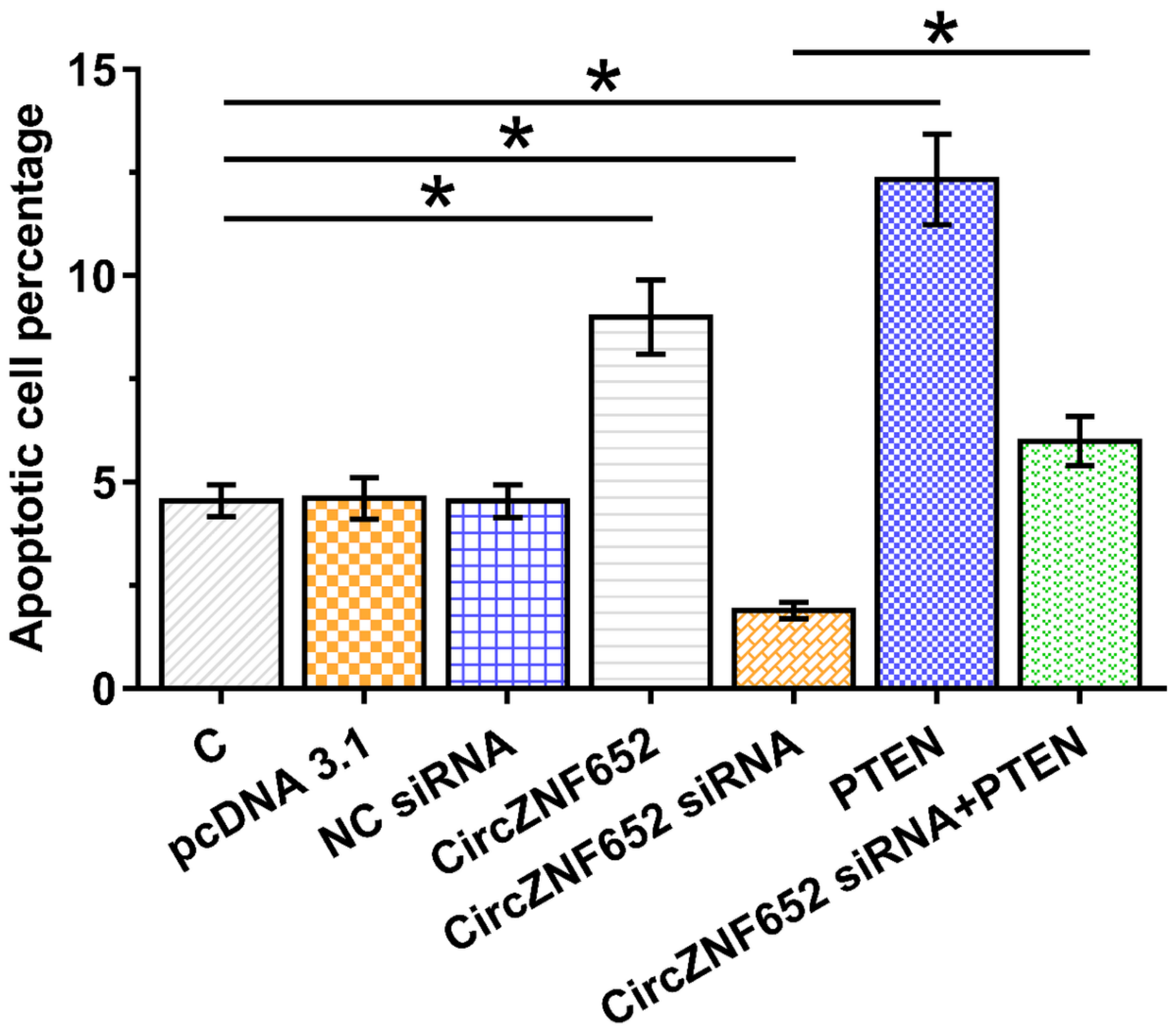

Figure 4

CircZNF652 positively regulated the apoptosis of chondrocytes induced by LPS through PTEN Chondrocytes were cultivated in medium containing $10 \mu \mathrm{g} / \mathrm{ml}$ LPS for further $48 \mathrm{~h}$ at $37^{\circ} \mathrm{C}$, followed by the analysis of cell apoptosis by cell apoptosis assay. Data of multiple cell transfection groups and LPS treatment groups was expressed as mean +/- SD values of three biological replicates. ${ }^{\star}, p<0.05$. 